\title{
Polymorphisms in IL10 may alter CD4 T-cell counts in Indonesian HIV patients beginning antiretroviral therapy
}

\author{
Deane Maria Dmello ${ }^{1}$, Ibnu Ariyanto ${ }^{2}$, Riwanti Estiasari ${ }^{2}$, Samuel Halstrom ${ }^{1}$, Jessica Gaff ${ }^{1}$, Silvia Lee ${ }^{1}$, \\ Patricia Price $^{1,2}$ \\ ${ }^{1}$ School of Biomedical Science, Curtin University, Perth, Western Australia, Australia \\ ${ }^{2}$ Faculty of Medicine, Universitas Indonesia, Jakarta, Indonesia
}

\section{Corresponding author}

A/Professor Patricia Price

School of Biomedical Science,

Curtin University,

Bentley 6102

Australia

Tel: 618-92669716

Email: patricia.price@curtin.edu.au

\section{Keywords}

Antiretroviral therapy, HIV, IL-10, single nucleotide polymorphism, CD4 T-cell counts, Indonesia

\section{Abbreviations}

ART: Antiretroviral therapy, IL: Interleukin, SNP: Single nucleotide polymorphism

\begin{abstract}
Interleukin 10 (IL-10) is a potent anti-inflammatory cytokine influenced by single nucleotide polymorphisms (SNP) located in upstream regulatory regions. Here we address the effects of five SNP (rs1518111, rs3021094, rs3024491, rs1800872 and rs1800871) on CD4 T-cell counts in Indonesian HIV patients assessed before ART and after 12 months on treatment. Heterozygosity at rs1518111 or rs1800872 associated with low CD4 T-cell counts at both time points. Both alleles were carried in two haplotypes. Haplotype $\underline{\mathbf{2}} 11 \underline{2} 2$ (present in $30 \%$ of participants) associated with low CD4 T-cell counts, whereas $\underline{\mathbf{2}} 12 \underline{2} 2$ (in $6 \%$ of participants) did not. Hence untyped SNP(s) tagged by $\underline{\mathbf{2}} 11 \underline{2} 2$ may depress CD 4 T-cell counts. The association with heterozygosity suggests synergy with an allele from a haplotype lacking rs1518111 and/or rs1800872.
\end{abstract}

(120 words) 


\section{Introduction}

CD4 T-cell counts are a reliable prognostic marker of HIV disease progression and risk of opportunistic infections. HIV-infected individuals differ in their rate of disease progression and their response to antiretroviral therapy (ART). This suggests complex interactions between the virus, the environment and the host genome. Host genes known to affect HIV disease include those encoding chemokine receptors, human leukocyte antigens (HLA), cytokines and apoptosis-related genes $(1,2)$.

Interleukin-10 (IL-10) is an anti-inflammatory and immunomodulatory cytokine produced by macrophages, monocytes, T-helper cells and B-lymphocytes. IL-10 can inhibit the production of proinflammatory cytokines and down-regulate the expression of HLA class I and II molecules. IL-10 production in humans is influenced by genetic factors, which consequently have potential to affect HIV disease. Previous studies have focused on single nucleotide polymorphisms (SNP) within the IL10 promoter region, notably -1082G/A (rs1800896), -592C/A (rs1800872) and -819C/T (rs1800871), and generate conflicting results. For example; the A allele of rs1800872 and the "ATA" haplotype defined by the three SNP increased susceptibility to HIV infection and disease progression in Indian cohorts $(3,4)$. However in an African population, carriers of AA at rs1800872 had attenuated CD4 T-cell loss and a broader CD8 T-cell response to HIV peptides, whilst rs1800896 more clearly affected plasma IL-10 levels (5). Similarly the A alleles were protective against HIV and Hepatitis B disease in an Estonian population using intravenous drugs (6). Studies from the Indian sub-continent associate -1082G/A (rs1800896) and 592C/A (rs1800872) with susceptibility to tuberculosis amongst HIV patients, but results included associations with heterozygous carriage (7).

This highlights the need for further studies to identify IL10 SNP that may affect CD4 T-cell counts before and on ART. A study of 21 SNP spanning IL10 in Caucasians HIV patients confirmed the -1082G/A ( $r$ 1800896), -592C/A ( $r$ 1800872) and -819C/T (rs1800871) haplotype as the most informative marker of disease progression but showed that it tagged and its effects were modified by broader IL1O haplotypes. Interestingly, no clear associations were found in African Americans (8).

The patterns may also be distinct in Asians, so we have investigated associations between five polymorphisms in IL10 (rs1518111, rs3021094, rs3024491, rs1800872 and rs1800871) and CD4 T-cell count recovery in HIV-infected Indonesian patients beginning ART. We sought the haplotypes that best define the risk. 


\section{Materials and Methods}

2.1 Patients and routine assessments: A longitudinal study of 79 HIV-1 patients was conducted at the HIV clinic at Cipto Mangunkusumo Hospital in Jakarta, Indonesia. Patients were invited to participate if they began ART with <200 CD4 T-cells/uL between March 2013 and March 2014. They were tested serologically for HCV, for oral candidiasis by clinical examination and for pulmonary tuberculosis by chest X-ray and sputum acid bacilli smear. The study was approved by the Ethics Committee of Cipto Mangunkusumo Hospital and Universitas Indonesia. Written informed consent was obtained from individuals who agreed to participate. CD4 T-cells were quantified by standard flow cytometry from the commencement of triple therapy (V0) including lamivudine, zidovudine, nevirapine, stavudine, efavirens and/or tenofovir. Most individuals were re-tested at 3, 6 and 12 months (V3, V6, V12). Reasons for discontinuation included death from causes related to AIDS (5 patients), drug-induced allergy requiring discontinuation of ART and loss to follow up. Plasma HIV RNA loads were determined using COBAS $^{\bullet}$ AmpliPrep/COBAS ${ }^{\circ}$ TaqMan $^{\circ}$ HIV-1 Tests (version 2.0).

2.2 Genotyping: DNA was quantified using a Qubit fluorometer (Thermo Fisher Scientific, Waltham, MA) and diluted to 50ng/uL. IL10 polymorphisms (rs1518111, rs3021094, rs3024491, rs1800872 and rs1800871) were genotyped using custom TaqMan OpenArray Genotyping Plates (Life Technologies, Grand Island, NY) (9). DNA samples were diluted at 1:1 in TaqMan OpenArray Genotyping Master Mix for 50 cycles of PCR amplification. The data was analyzed using the OpenArray SNP Genotyping Analysis software, and the genotypes were assigned manually. Chi Square analysis with $5 \%$ significance level and 1 degree of freedom was used to determine if the observed allele frequencies were in Hardy-Weinberg equilibrium (HWE). Haplotypes and their estimated frequencies were calculated using the fastPHASE algorithm with the default parameters and sampled from the observed genotypes 10,000 times (10). Haplotypes with an estimated frequency $<1 \%$ were excluded.

2.3 Statistical Analyses: Statistical analyses were done with GraphPad Prism software (v6; Tree Star, La Jolla, CA), using non-parametric Mann-Whitney tests, Chi $^{2}$ or Fisher's exact tests. A $5 \%$ significance level $(P<0.05)$ was considered statistically significant.

\section{Results and Discussion}

Seventy-nine Indonesian HIV-infected patients were screened for IL10 polymorphisms and their effect on CD4 T-cell counts before ART and after 12 months on treatment. The cohort included 51(65\%) males and had a median (range) age of 31(19-48) years. The CD4 T-cell count at baseline was 61(3-199) cells/ul. $37(47 \%)$ of the patients had pulmonary tuberculosis and $17(22 \%)$ were co-infected with Hepatitis C. 
Heterosexual transmission accounted for 46 subjects (58\%), with homosexual transmission and intravenous drug use each accounting for $18 \%$. This is a common pattern in many Asian centres.

No genotypes associated with pulmonary tuberculosis, candidiasis or HCV (data not shown). Table 1 summarises associations between the five IL10 SNP genotypes and CD4 T-cell counts. As the IL10 rs3024491 $(2,2)$ genotype was rare, patients with this genotype were analysed with those carrying rs3024491 (1,2). Univariate analyses associated heterozygous carriage of the minor alleles of two SNP with CD4 T-cell counts recorded before ART ( $r$ 1518111; $p=0.02$ and $r s 1800872 ; p=0.03$ ) and after 3, 6 and 12 months (see Table 1), so the effect remains evident on ART. rs1518111 and rs1800872 are in linkage disequilibrium (1000 Genomes, $\left.D^{\prime}=1, R^{2}=0.925\right)$ in East Asians and Europeans, so common associations are plausible.

The $\mathrm{G}$ allele of the commonly studied -1082G/A (rs1800896) was also checked and found to be rare in our Indonesian cohort (MAF=0.09). Moreover it is not in linkage disequilibrium with rs1800872 in East Asians described in the 1000 genomes database. Accordingly, carriage of the minor (G) allele of rs1800896 did not affect CD4 T-cell counts before ART or after 12 months (data not shown, $p=0.77-0.83$ ).

To resolve the effect of heterozygous carriage of rs1518111 and rs1800872 and address which SNP were responsible for the phenotype, we considered the haplotypes carried by individuals in the cohort. Fifteen haplotypes of the five IL10 SNP described in Table 1 were derived. Five haplotypes occurred at an estimated frequency of $1 \%$ or greater and accounted for $94 \%$ of this population (Table 2). The alleles of the SNP in each haplotype are expressed as a 1 (major allele) or a 2 (minor allele), in chromosomal order as listed in Table 1. Carriage of the haplotype (lllllll $\left.1 \begin{array}{lll}\mathbf{2} & \mathbf{2} & 2\end{array}\right)$ was associated with low CD4 T-cell counts $(p=0.02$, Table 2). This haplotype contains the minor alleles of three SNP, where the first ( $r$ 1518111) and fourth (rs1800872) were associated with CD4 T-cell counts (Table 1). These two minor alleles also occurred in a rarer haplotype $\left(\begin{array}{lllll}\mathbf{2} & 1 & \mathbf{2} & \underline{\mathbf{2}} & 2\end{array}\right)$ that showed no association with low CD4 T-cell counts. This suggests that neither SNP is directly responsible for the low CD4 T-cell counts - rather the effect appears

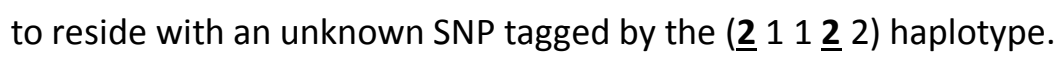

In conclusion; our data links heterozygosity at IL10 rs1518111 and rs1800872 with low CD4 T-cell counts in untreated HIV-infected Indonesian patients and on ART. Derivation of 5-SNP haplotypes demonstrated that these SNP are not directly responsible for the effect on CD4 T-cell counts. Moreover the consistent association with heterozygosity suggests synergy between SNP carried in distinct haplotypes, perhaps including the distal promoter region (8). As data in the 1000 Genomes database show distinct IL10 haplotypes carried by different ethnic groups, studies of CD4 T-cell recovery in other ethnicities may reveal which SNP are critical. As IL-10 is critical for limiting inflammation and the host immune response to pathogens, the association with IL10 haplotypes confirms that these pathways influence CD4 T-cell loss and recovery on ART. 


\section{Acknowledgements}

The authors thank patients who participated in our study and Ms Faizah for co-ordinating their visits and assembling the database. The project was supported by Universitas Indonesia and Curtin University.

\section{References}

1. Qin M, Brummel S, Singh KK, Fenton T, Spector SA. Associations of host genetic variants on CD4 lymphocyte count and plasma HIV-1 RNA in antiretroviral naive children. Ped Infect Dis Journal. 33 (2014):946-52.

2. Haas DW, Geraghty DE, Andersen J, Mar J, Motsinger AA, D'Aquila RT, et al. Immunogenetics of CD4 lymphocyte count recovery during antiretroviral therapy: An AIDS Clinical Trials Group study. J Infect Dis. 194 (2006):1098-107.

3. Chatterjee A, Rathore A, Sivarama P, Yamamoto N, Dhole TN. Genetic association of IL-10 gene promoter polymorphism and HIV-1 infection in North Indians. J ClinImmunol. 29 (2009):71-7.

4. Singh S, Sharma A, Arora SK. Combination of low producer AA-genotypes in IFN-gamma and IL-10 genes makes a high risk genetic variant for HIV disease progression. Cytokine. 77 (2016):135-44.

5. Naicker DD, Wang B, Losina E, Zupkosky J, Bryan S, Reddy S, et al. Association of IL-10-promoter genetic variants with the rate of CD4 T-cell loss, IL-10 plasma levels, and breadth of cytotoxic T-cell lymphocyte response during chronic HIV-1 infection. Clin Infect Dis 54 (2012):294-302.

6. Kallas E, Huik K, Pauskar M, Jogeda EL, Karki T, Des Jarlais D, et al. Influence of interleukin 10 polymorphisms -592 and -1082 to the HIV, HBV and HCV serostatus among intravenous drug users. Infect Genet Evolution. 30 (2015):175-80.

7. Ramaseri Sunder S, Hanumanth SR, Nagaraju RT, Venkata SK, Suryadevara NC, Pydi SS, et al. IL10 high producing genotype predisposes HIV infected individuals to TB infection. Human Immunol. 73 (2012):605-11.

8. Oleksyk TK, Shrestha S, Truelove AL, Goedert JJ, Donfield SM, Phair J, et al. Extended IL10 haplotypes and their association with HIV progression to AIDS. Genes Immun. 10 (2009):309-22.

9. Goullee H, Wadley AL, Cherry CL, Allcock RJ, Black M, Kamerman PR, et al. Polymorphisms in CAMKK2 may predict sensory neuropathy in African HIV patients. J Neurovirol. 22 (2016):508-17.

10. Scheet P, Stephens M. A fast and flexible statistical model for large-scale population genotype data: applications to inferring missing genotypes and haplotypic phase. Am J Hum Gen. 78(2006):629-44. 
Table 1: Two IL10 SNPs associated with low CD4 T-cell counts before and on ART.

\begin{tabular}{|c|c|c|c|c|c|c|c|}
\hline \multirow[t]{2}{*}{ SNP ID } & \multirow{2}{*}{$\begin{array}{c}\text { V0/V3 } \\
\text { V6/V12 }^{\mathrm{a}}\end{array}$} & \multicolumn{3}{|c|}{ CD4 T-cell counts ${ }^{b}$} & \multicolumn{3}{|c|}{ P-value ${ }^{c}$} \\
\hline & & 1,1 & 1,2 & 2,2 & 1,1 vs 1,2 & 1,1 vs 2,2 & 1,2 vs 2,2 \\
\hline \multirow{8}{*}{$\begin{array}{c}\text { rs1518111 } \\
C(0.40)^{d}\end{array}$} & vo & 72(9-199) & $48(3-191)$ & $74(2-187)$ & 0.02 & 0.18 & 0.91 \\
\hline & & $(n=30)$ & $(n=30)$ & $(n=15)$ & & & \\
\hline & V3 & $212(79-601)$ & $169(7-492)$ & $148(11-410)$ & 0.08 & 0.04 & 0.50 \\
\hline & & $(n=28)$ & $(n=25)$ & $(n=14)$ & & & \\
\hline & V6 & $226(118-516)$ & $173(6-501)$ & $221(20-394)$ & 0.04 & 0.31 & 0.83 \\
\hline & & $(n=26)$ & $(n=23)$ & $(n=13)$ & & & \\
\hline & V12 & $304(171-763)$ & $242(121-616)$ & $328.5(44-736)$ & 0.057 & 0.72 & 0.67 \\
\hline & & $(n=24)$ & $(n=21)$ & $(n=12)$ & & & \\
\hline \multirow{4}{*}{$\begin{array}{c}\text { rs3021094e } \\
\text { G }(0.46)\end{array}$} & vo & $49(2-187)$ & $65(3-198)$ & 69(9-199) & 0.72 & 0.12 & 0.26 \\
\hline & & $(n=24)$ & $(n=26)$ & $(n=19)$ & & & \\
\hline & V12 & $283.5(44-736)$ & $285.5(121-603)$ & $304(171-763)$ & 0.87 & 0.5 & 0.32 \\
\hline & & $(n=18)$ & $(n=20)$ & $(n=16)$ & & & \\
\hline \multirow{4}{*}{$\begin{array}{c}\text { rs3024491 }^{f} \\
A(0.06)\end{array}$} & vo & $62(2-199)$ & $94(4-191)$ & - & 0.49 & - & - \\
\hline & & $(n=68)$ & $(n=8)$ & & & & \\
\hline & V12 & $282(44-763)$ & 377(101-496) & - & 0.38 & - & - \\
\hline & & $(n=53)$ & $(n=6)$ & & & & \\
\hline \multirow{8}{*}{$\begin{array}{c}\text { rs1800872 } \\
G(0.42)\end{array}$} & vo & 69(9-199) & $45(3-191)$ & $52(2-187)$ & 0.03 & 0.1 & 0.83 \\
\hline & & $(n=29)$ & $(n=32)$ & $(n=16)$ & & & \\
\hline & V3 & $206(79-601)$ & $136(7-492)$ & 159(11-410) & 0.03 & 0.08 & 0.80 \\
\hline & & $(n=27)$ & $(n=27)$ & $(n=15)$ & & & \\
\hline & V6 & $242(118-516)$ & $157(6-501)$ & $226(20-394)$ & 0.02 & 0.45 & 0.39 \\
\hline & & $(n=25)$ & $(n=25)$ & $(n=14)$ & & & \\
\hline & V12 & $304(171-763)$ & $207(121-616)$ & 298(44-736) & 0.01 & 0.67 & 0.41 \\
\hline & & $(n=23)$ & $(n=23)$ & $(n=13)$ & & & \\
\hline \multirow{4}{*}{$\begin{array}{c}\text { rs1800871 } \\
\text { G (0.43) }\end{array}$} & vo & $67(2-187)$ & $48(3-198)$ & $67(9-196)$ & 0.21 & 0.26 & 0.82 \\
\hline & & $(n=16)$ & $(n=29)$ & $(n=26)$ & & & \\
\hline & V12 & $286.5(144-763)$ & $233.5(121-616)$ & $328.5(44-736)$ & 0.21 & 0.96 & 0.79 \\
\hline & & $(n=22)$ & $(n=22)$ & $(n=12)$ & & & \\
\hline
\end{tabular}

a. V0: CD4 T-cell counts before ART. V3, V6 and V12: CD4 T-cell counts after 3, 6 and 12 months on ART

b. Median (range) CD4 T-cell counts (cells/ul)

c. Non-parametric Mann-Whitney test analysing association between the IL10 genotypes and CD4 T-cell counts $(\mathrm{P}<0.05)$

d. Minor allele (MAF), where MAF are calculated from samples genotyped successfully

e. Observed allele frequencies deviated from the expectations of $\operatorname{HWE}\left(\left(X^{2} 4.01>X^{2} 0.05,1 d f(3.84)\right)\right.$

f. $(1,2)$ and $(2,2)$ were merged as $<5$ individuals carried the $(2,2)$ genotype 
Table 2: One haplotype associated with low CD4 T-cell counts before and on ART

\begin{tabular}{|c|c|c|c|c|c|c|c|}
\hline \multirow{2}{*}{$\begin{array}{l}\text { Haplotype } \\
\text { Sequence }^{\mathrm{a}}\end{array}$} & \multirow{2}{*}{ V0/V12 } & \multicolumn{3}{|c|}{ CD4 T-cell counts ${ }^{c}$} & \multicolumn{3}{|c|}{ P-value $^{d}$} \\
\hline & & 0 & 1 & 2 & 1 vs 2 & 0 vs 2 & 0 vs 1 \\
\hline \multirow{2}{*}{$\begin{array}{l}12111 \\
(0.41)^{b}\end{array}$} & Vo & $\begin{array}{c}50(2-198) \\
(n=31)\end{array}$ & $\begin{array}{c}62(3-196) \\
(n=27)\end{array}$ & $\begin{array}{c}\text { 69(9-199) } \\
(n=21)\end{array}$ & 0.17 & 0.12 & 0.81 \\
\hline & V12 & $\begin{array}{c}253(44-736) \\
(n=24)\end{array}$ & $\begin{array}{c}289(121-567) \\
(n=20)\end{array}$ & $\begin{array}{c}304(171-763) \\
(n=17)\end{array}$ & 0.46 & 0.21 & 0.75 \\
\hline \multirow{2}{*}{$\begin{array}{c}21122 \\
(0.3)\end{array}$} & Vo & $\begin{array}{c}72(7-199) \\
(n=38)\end{array}$ & $\begin{array}{c}48(3-180) \\
(n=30)\end{array}$ & $\begin{array}{c}50(2-166) \\
(n=11)\end{array}$ & 0.72 & 0.07 & 0.02 \\
\hline & V12 & $\begin{array}{c}310(144-763) \\
(n=29)\end{array}$ & $\begin{array}{c}207(101-616) \\
(n=23)\end{array}$ & $\begin{array}{c}285(44-736) \\
(n=9)\end{array}$ & 0.48 & 0.61 & 0.003 \\
\hline \multirow{2}{*}{$\begin{array}{l}11111 \\
(0.14)\end{array}$} & Vo & $\begin{array}{c}63(2-199) \\
(n=62)\end{array}$ & $\begin{array}{c}55(8-198) \\
(n=17)\end{array}$ & - & - & - & 0.61 \\
\hline & V12 & $\begin{array}{c}288(44-763) \\
(n=49)\end{array}$ & $\begin{array}{c}276(141-616) \\
(n=12)\end{array}$ & - & - & - & 1.0 \\
\hline \multirow{2}{*}{$\begin{array}{l}21222 \\
(0.06)\end{array}$} & Vo & $\begin{array}{c}55(2-199) \\
(n=71)\end{array}$ & $\begin{array}{c}94(4-191) \\
(n=8)\end{array}$ & - & - & - & 0.44 \\
\hline & V12 & $\begin{array}{c}282(44-763) \\
(n=55)\end{array}$ & $\begin{array}{c}377(101-496) \\
(n=6)\end{array}$ & - & - & - & 0.35 \\
\hline \multirow{2}{*}{$\begin{array}{c}12112 \\
(0.3)\end{array}$} & vo & $\begin{array}{c}55(2-199) \\
(n=75)\end{array}$ & $\begin{array}{c}144(21-198) \\
(n=4)\end{array}$ & - & - & - & 0.17 \\
\hline & V12 & $\begin{array}{c}285(44-763) \\
(n=58)\end{array}$ & $\begin{array}{c}441(144-603) \\
(n=3)\end{array}$ & - & - & - & 0.50 \\
\hline
\end{tabular}

a. Defined by SNP alleles in the order shown in Table 1

b. Haplotype frequencies determined using the most probable assignment for each individual

c. Median (range) CD4 T-cells/ul. 0, 1 and 2 represent patients without the haplotype or with 1 or 2 copies

d. Non-parametric Mann-Whitney test 